\title{
Associations between Genetic Variants in the NOS1AP (CAPON) Gene and Cardiac Repolarization in the Old Order Amish
}

\author{
Wendy Post ${ }^{\mathrm{a}, \mathrm{b}}$ Haiqing Shen ${ }^{c}$ Coleen Damcott ${ }^{c}$ Dan E. Arking ${ }^{\mathrm{a}, \mathrm{d}}$ \\ W.H. Linda Kao ${ }^{b}$ Paul A. Sack ${ }^{c}$ Kathleen A. Ryan ${ }^{c}$ Aravinda Chakravarti $^{d}$ \\ Braxton D. Mitchell ${ }^{c}$ Alan R. Shuldiner ${ }^{c, e}$

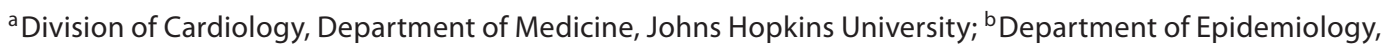 \\ Johns Hopkins University Bloomberg School of Public Health; ' Division of Endocrinology, Diabetes, and Nutrition, \\ Department of Medicine, University of Maryland; dMcKusick-Nathans Institute of Genetic Medicine, \\ Johns Hopkins University School of Medicine, and 'Geriatrics Research and Education Clinical Center, \\ Baltimore Veterans Administration Medical Center, Baltimore, Md., USA
}

\section{Key Words}

Association analysis - Candidate gene analysis •

Cardiovascular diseases - Genetic polymorphisms .

Heritability $\cdot$ Isolated population

\begin{abstract}
Background: Through a genome-wide association study, we discovered an association of the electrocardiographic QT interval with polymorphisms in the NOS1AP (CAPON) gene. The purpose of the current study was to replicate this association in the Old Order Amish. Methods: Four NOS1AP SNPs were selected that captured all major haplotypes in the region of interest ( $\sim 120 \mathrm{~kb}$ segment). Genotyping was completed in 763 subjects from the Heredity and Phenotype Intervention (HAPI) Heart Study. Association analyses were performed using a variance components methodology, accounting for relatedness of individuals. Results: Heritability of the QT interval was $0.50 \pm 0.09\left(p=1.9 \times 10^{-9}\right)$. All four SNPs were common with a high degree of correlation between SNPs. Two of the four SNPs (pairwise $r^{2}=0.86$ ) were significantly associated with variation in adjusted QT inter-
\end{abstract}

val (rs1415262, $p=0.02$ and $r s 10494366, p=0.006$, additive models for both). SNP rs10494366 explained $0.9 \%$ of QT interval variability, with an average genetic effect of $6.1 \mathrm{~ms}$. Haplotypes that contained the minor allele for rs10494366 were associated with longer QT interval. Conclusions: This study provides further evidence that NOS1AP variants influence QT interval and further validates the utility of genomewide association studies, a relatively new approach to gene discovery.

Copyright $\odot 2007$ S. Karger AG, Basel

\section{Introduction:}

The electrocardiographic QT interval is a measure of cardiac repolarization which is moderately heritable $[1,2]$ and predicts risk for cardiovascular events in the general population [3-5]. Long QT syndrome is a rare heterogeneous single gene disorder caused by mutations in one of a variety of ion channel genes, which can lead to fatal arrhythmias [6]; however, the majority of sudden arrhythmic deaths occur in people without mutations in these

\section{KARGER}

Fax +4161306 1234 E-Mail karger@karger.ch www.karger.com
(C) 2007 S. Karger AG, Basel 0001-5652/07/0644-0214\$23.50/0

Accessible online at:

www.karger.com/hhe
Wendy Post, MD, MS

Division of Cardiology, Department of Medicine, Johns Hopkins University

Blalock 910H, 600 N. Wolfe Street

Baltimore, MD 21287 (USA)

Tel. +1 410955 7376, Fax +1 443287 0121, E-Mail wpost@jhmi.edu 
genes [7]. Indeed, determinants of the QT interval in the general population and the risk for sudden arrhythmic cardiac death are complex, and are determined by genetic background, the environment, and their interactions.

We previously undertook a three-stage genome-wide association study, designed to identify genes or genomic regions that modulate the QT interval, by initially genotyping $\sim 115,000$ single nucleotide polymorphisms (SNPs) in 100 subjects from each of the extremes of the QT interval distribution (total 200 subjects) in the KORA S4 Study ('Cooperative Research in the Region of Augsburg'), a population based epidemiological study in Augsburg, Germany [8]. We identified and replicated an association between QT interval with SNPs in Nitric Oxide Synthase 1 (Neuronal) Adaptor Protein (NOS1AP also known as CAPON) on chromosome 1 , which is the carboxy-terminal PDZ ligand of neuronal nitric oxide synthase (nNOS).

The purpose of the current study was to evaluate, and replicate, the association between NOS1AP and the QT interval in a more genetically homogenous population, the Old Order Amish, and to estimate the heritability of the QT interval. The Old Order Amish of Lancaster County, Pennsylvania are a rural, closed, genetically isolated population who are descendents from a limited number of individuals who emigrated from Europe in the 1700 's. They eschew modern technology, including many standard medical therapies, and live a relatively homogeneous, active lifestyle [9]. There are potential advantages to performing genetic association studies in the Amish. Genetic association studies in isolated populations are less likely to be influenced by spurious associations due to population substructure, and there are fewer non-genetic factors that could potentially confound associations between complex traits and genetic polymorphisms due to their relative environmental homogeneity.

Although the association between NOS1AP and the QT interval was replicated in our initial report, replication in multiple populations helps to confirm that the association is valid in these populations that have the potential for different genetic backgrounds and environmental influences that have the potential for different genetic backgrounds and environmental influences that could negate or otherwise modify the main effect of the gene variant. Moreover, if heterogeneity in associations is observed in different populations, assuming that the differences were not due to study design issues, our understanding of the role of these genetic variations in different populations may be refined or additional insight into the potential interactions between genetic variations and other underlying risk factors in various populations may be gleaned [10]. Further validation of our genome-wide association study results would extend the association between NOS1AP and the QT interval to additional populations, and also strengthen the evidence for the potential utility of genome-wide association studies, a relatively new approach to gene discovery.

\section{Methods}

\section{Subjects/Phenotyping}

The subjects in this study were Amish men and women, enrolled between 2003 and 2006 in the Heredity and Phenotype Intervention (HAPI) Heart Study, which was designed to identify genes that interact with the environment to influence cardiovascular risk. Subjects were generally healthy and were initially recruited into the HAPI Heart Study on the basis of their prior participation in one of our previous studies of bone mineral density and/or coronary calcification, although for practical considerations (e.g., to maintain rapport with the community and to more efficiently meet recruitment goals), recruitment guidelines were modified to allow other interested individuals in the community to participate. These subjects were initially identified through word of mouth, advertisements, Amish-wide mailings, and referrals from local physicians. Participating subjects were aged 20 years and older, and their first and second degree relatives were encouraged to participate. Subjects with severe hypertension (BP $>180 / 105 \mathrm{~mm} \mathrm{Hg}$ ), malignancy, kidney, liver or uncontrolled thyroid disease were not eligible for the study.

All subjects underwent a research examination at the Amish Research Clinic in Strasburg, PA. This examination included a 12-lead electrocardiogram (ECG) which was acquired using standard methods with a GE Marquette digital recording system. The QT measurements were calculated by the Marquette computer system using 'median beats'. There were 12 median beats, one for each of the 12 leads, which was developed using simultaneously sampled data, allowing for them to be time aligned synchronously. A vector magnitude signal was formed by combining all 12lead median beats, so-called 'superbeat'. The QT interval was calculated from the superbeat, which measures the earliest onset of the QRS to the latest offset of the T wave across all 12 leads.

The analyses described in this report are based on 797 subjects recruited into the HAPI Heart Study with at least one genotype in the NOS1AP gene available. We excluded one subject with atrial fibrillation and 33 subjects with a QRS duration $\geq 120 \mathrm{~ms}$, as these conditions can alter repolarization (QT interval). Our final analysis set included 763 subjects.

The research protocols were approved by the Institutional Review Boards of the University of Maryland and the Johns Hopkins University. All subjects signed informed consent, which included permission to perform genotyping.

\section{SNP Selection and Genotyping}

Four SNPs in NOS1AP (rs4657139, rs12567211, rs1415262, rs10494366) were selected for genotyping based on our previous work in the KORA Study. In stage II of that study, which consisted of 300 individuals from each extreme of the QT interval dis- 
Table 1. Characteristics of the Amish HAPI Heart Study population

\begin{tabular}{lccc}
\hline Characteristics (mean \pm SD) & $\begin{array}{l}\text { Total } \\
(\mathrm{n}=763)\end{array}$ & $\begin{array}{l}\text { Men } \\
(\mathrm{n}=402)\end{array}$ & $\begin{array}{l}\text { Women } \\
(\mathrm{n}=361)\end{array}$ \\
\hline Age, years & $43.5 \pm 14.1$ & $41.6 \pm 13.5$ & $45.5 \pm 14.4$ \\
Mean QT interval, ms & $409.5 \pm 28.7$ & $411.3 \pm 29.0$ & $407.4 \pm 28.4$ \\
Adjusted QT interval*, ms & $408.8 \pm 29.2$ & $411.4 \pm 28.5$ & $407.9 \pm 28.2$ \\
${\text { Body mass index, } \mathrm{kg} / \mathrm{m}^{2}}^{2}$ & $26.6 \pm 4.5$ & $25.6 \pm 3.2$ & $27.6 \pm 5.4$ \\
Systolic blood pressure, $\mathrm{mm} \mathrm{Hg}$ & $121.1 \pm 14.9$ & $121.2 \pm 12.7$ & $121.0 \pm 17.0$ \\
Diastolic blood pressure, $\mathrm{mm} \mathrm{Hg}$ & $76.6 \pm 8.8$ & $77.6 \pm 9.0$ & $75.6 \pm 8.5$ \\
\hline
\end{tabular}

* Adjusted for age, gender, heart rate and family structure. tribution (total of 600 individuals), fine mapping had revealed that the $5^{\prime}$ end of the gene, and in particular, a $\sim 120-\mathrm{kb}$ segment exhibiting strong linkage disequilibrium (LD), displayed the greatest association with QT interval [8]. Using the Tagger tool in Haploview (http://www.broad.mit.edu/mpg/haploview/) these four common SNPs capture all major haplotypes in this region ( $>1 \%$ frequency). To estimate coverage, using an $r^{2}$ of 0.5 in the HapMap CEU samples, we capture 44 of the 89 alleles with a minor allele frequency $>0.05$ in this region. Seventy-three percent of the captured alleles have an $r^{2}>0.8$. Genotyping was performed using TaqMan Assays on Demand or Assays by Design (Applied Biosystems) according to manufacturer's protocols.

\section{Statistical Methods}

Genotypes were checked for Mendelian consistency using the PedCheck software program in the extended Amish pedigree. Allele frequencies were calculated for each SNP by gene counting, and observed genotypes were tested for fit to the expectations of the Hardy-Weinberg law using the $\chi^{2}$ test. Pairwise LD $\left(\mathrm{r}^{2}\right)$ was estimated by Haploview. Heritability and association analyses were performed using a variance components methodology to account for the relatedness of study subjects as implemented in the Sequential Oligogenic Linkage Analysis Routines (SOLAR) program [11]. We modeled variation in QT interval as a function of measured environmental covariates, additive genetic effects and a residual error component. The additive genetic effects were parameterized as a function of the kinship matrix. Maximum likelihood methods were used to estimate the covariate and genetic effects simultaneously. Initially, we estimated the heritability of QT interval as the proportion of the total phenotypic variation that could be attributable to additive genetic effects. We then evaluated the association between NOS1AP genotype and QT interval while simultaneously adjusting for the effects of age, age ${ }^{2}$, sex, and heart rate and additive genetic effects (heritability). Statistical significance of the genotype effects were assessed using the likelihood ratio test, in which we compared the likelihood of the data under a model in which the genotype effect was estimated against the likelihood of a nested model in which the genotype effect was constrained to zero. Analyses were performed assuming an additive genetic model. Since some associations appeared to follow a dominant pattern, we repeated the analyses assuming a dominant model. We evaluated gene-by-gender interactions for each SNP using the log likelihood ratio test comparing the full model with a gene* gender term and a nested model without the gene* ${ }^{*}$ gender term. We estimated that a sample of size 763 subjects provides $80 \%$ power to identify SNPs accounting for as little as $1 \%$ of the variation in QT interval, with an alpha level of 0.05 and minor allele frequency of 0.30 . Haplotype frequencies and association were calculated based on the Stochastic_EM (SEM) algorithm using THESIAS program [12].

\section{Results}

The characteristics of the study population are described in table 1 . The sample size of 763 subjects includes individuals from 466 sibships, with sibships ranging in size from 1 to 9 . Additional relationship types were identified by linking study subjects into larger pedigrees through their examined or unexamined parents. These 763 individuals could be combined into 377 multiplex pedigrees, ranging in size from 1 to 35 examined individuals and representing 466 sibpairs, 241 parent-offspring pairs, 352 avuncular pairs and 156 first-cousin pairs.

The mean ( \pm SD) age was $44 \pm 14$ years and $47 \%$ were women. QT was normally distributed (skewness $=0.43$ and kurtosis $=0.73$ ) with mean $( \pm$ SD) of $409.5 \pm$ $28.7 \mathrm{~ms}$. Heritability of the QT interval, adjusted for age, gender and heart rate, was estimated at $0.50 \pm 0.09(\mathrm{p}=$ $\left.1.9 \times 10^{-9}\right)$. The proportion of the variance due to the covariates (age, age ${ }^{2}$, gender, and heart rate) was 0.63 . All four SNPs were relatively common with minor allele frequencies between 34 and $45 \%$ and satisfied HardyWeinberg equilibrium. There was a high degree of correlation between SNPs (pairwise $\mathrm{r}^{2}$ ranging from 0.62 to 0.86 ) as displayed in figure 1.

Two of the four NOS1AP SNPs were significantly associated with variation in adjusted QT interval, as shown in table 2 ( $r s 1415262, \mathrm{p}=0.02$, additive model; $\mathrm{p}=0.008$, dominant model; and rs10494366, $\mathrm{p}=0.006$, additive model; $\mathrm{p}=0.02$, dominant model). Of note, the $\mathrm{r}^{2}$ of pair- 
Table 2. Associations between NOS1AP SNPs and adjusted QT interval

\begin{tabular}{|c|c|c|c|c|c|c|}
\hline \multirow[t]{2}{*}{ NOS1AP SNP (N/n) } & \multirow[t]{2}{*}{ MAF } & \multicolumn{3}{|c|}{ Adjusted QT interval* } & \multirow{2}{*}{$\begin{array}{l}\text { Additive } \\
\text { model } \\
\text { p value* }\end{array}$} & \multirow{2}{*}{$\begin{array}{l}\text { Dominant } \\
\text { model } \\
\text { p value* }\end{array}$} \\
\hline & & $\mathrm{NN}$ & $\mathrm{Nn}$ & $\mathrm{nn}$ & & \\
\hline rs4657139 (A/T) & 0.37 & $408.4 \pm 1.3$ & $411.0 \pm 1.5$ & $411.8 \pm 2.2$ & 0.1 & 0.05 \\
\hline rs12567211 (C/A) & 0.34 & $409.1 \pm 2.4$ & $410.7 \pm 2.3$ & $409.3 \pm 2.2$ & 0.84 & 0.45 \\
\hline rs1415262 (G/C) & 0.41 & $407.2 \pm 1.4$ & $410.6 \pm 1.4$ & $412.1 \pm 2.1$ & 0.02 & 0.008 \\
\hline rs10494366 (T/G) & 0.45 & $407.2 \pm 1.4$ & $409.9 \pm 1.5$ & $413.3 \pm 2.0$ & 0.006 & 0.02 \\
\hline
\end{tabular}

$\mathrm{N}=$ Major allele; $\mathrm{n}=$ minor allele. MAF is minor allele frequency.

* Adjusted for age, gender, heart rate, and family structure.

wise linkage disequilibrium between these two SNPs was 0.86 . There was a trend towards an association between rs4657139 and adjusted QT interval ( $\mathrm{p}=0.05$, dominant model). There were no associations between rs12567211 and the QT interval in single SNP analyses. There were no significant gene-by-gender interactions. SNP rs10494366 explained $0.9 \%$ of the variability in the QT interval, and the average genetic effect, measured as the difference in the mean QT intervals between the two homozygote genotypes, was $6.1 \mathrm{~ms}$.

We also performed haplotype analyses using these 4 SNPs. There were 2 major haplotypes in this Amish cohort. One haplotype was comprised of all the major alleles (1111) for these 4 SNPs (haplotype frequency 55\%) and the other of all the minor alleles (2222) (haplotype frequency $33 \%)$. There were three additional haplotypes $(2121,1121,2221)$ that were each present in $3-4 \%$ of the sample (note the order of the 4 SNPs is the same as in table 2). Consistent with the single SNP analyses, haplotypes that contained the minor allele for rs10494366 were associated with longer QT interval.

\section{Discussion}

Sudden unexpected cardiac death, generally attributed to a lethal arrhythmia, accounts for nearly $50 \%$ of coronary heart disease mortality [7] and has a familial component [13]. Abnormalities in cardiac repolarization, which can increase risk for arrhythmias, can manifest on a 12-lead electrocardiogram as an alteration in the QT interval. Little is known about specific genes associated with risk for sudden cardiac death in the general population, other than a few candidate gene studies [14-16]. We found that the QT interval is a moderately heritable trait

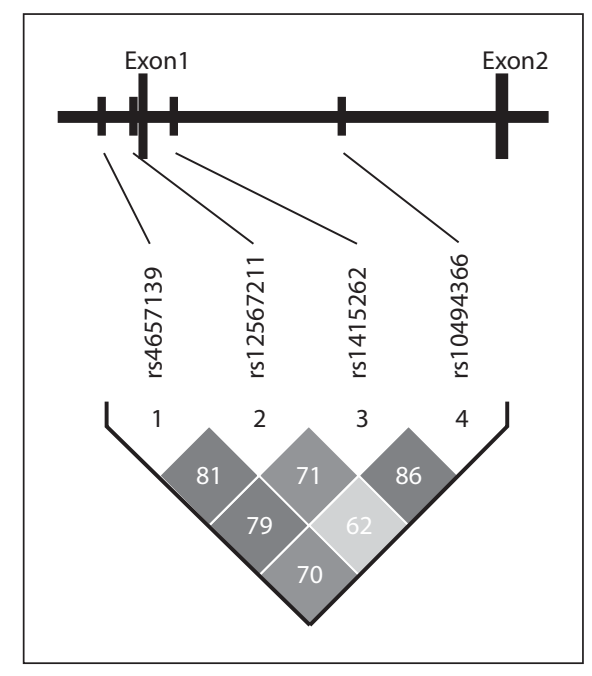

Fig. 1. $r^{2}$ plot of pairwise linkage disequilibrium between the four SNPs that cover a $120-\mathrm{kb}$ region in the $5^{\prime}$ portion of the NOS1AP gene.

$\left(\mathrm{h}^{2}=0.50 \pm 0.09\right)$ in the Old Order Amish. Our heritability results are slightly higher than the heritability estimates in the Framingham Heart Study $\left(\mathrm{h}^{2}=0.35\right)[2]$ and the Family Heart Study $\left(h^{2}=0.34\right)$ [17].

We have further validated the association between variants in the NOS1AP (CAPON) gene and the QT interval, which was recently discovered in a genome-wide association study [8]. Remarkably, we find very similar results in the Old Order Amish, a genetically isolated population, and the results seen in the German KORA Study [8]. In the Amish, the proportion of the variability in the QT interval that was explained by SNP rs 10494366 was $0.9 \%$ and the average genetic effect, measured as the difference in the mean QT intervals between the two ho- 
mozygote genotypes, was 6.1 ms. In KORA S4 and KORA F3, two distinct samples from a population-based German cohort, the proportion of the variability explained by the same allele of the same SNP was 1.2 and $1.9 \%$, respectively, and the average genetic effects were 4.9 and $7.9 \mathrm{~ms}$, respectively. The frequency of the minor allele was somewhat higher in the Amish, 45 versus $36 \%$ in the KORA S4 and F3 populations, and 39\% in the Framingham Heart Study, in which association between NOS1AP and QT interval was also shown [8]. A limitation of this study in the Old Order Amish is the smaller sample size (763 subjects versus 2,000-4,000 in the previous studies), leading to somewhat less power to detect associations and therefore less significant $\mathrm{p}$ values; however, the importance of these results is reflected in the remarkable consistency of the point estimates between this study and the others, suggesting that these findings are likely true positives.

We have added significantly to the previous study by performing these analyses in a genetically isolated population, in which spurious associations due to population substructure are less likely. We have also performed haplotype analyses, utilizing 4 SNPs that were identified to tag the $120-\mathrm{kb}$ region at the $5^{\prime}$ region of the gene, which is likely to harbor the functional variant. Since there was a high degree of LD between these SNPs in the Old Order Amish, the haplotype analysis did not provide significant additional information, but did suggest that haplotypes containing the minor allele for rs10494366 are most associated with longer QT intervals. Most likely, the functional variant is in a non-coding region with regulatory function, since sequencing in KORA did not identify any missense mutations in the exons [8].

It is remarkable that when comparing the results across the various cohort studies, findings are relatively consistent even though each study used a different algorithm for measuring QT interval. At this time, there is no gold standard for population studies, and the association persists regardless of the QT measurement method utilized.

In summary, we have further validated the discovery that the gene NOS1AP (CAPON) is associated with modulation of cardiac repolarization as manifested on the surface electrocardiogram in a genetically isolated homogeneous population. The trait is moderately heritable, and the functional variant is likely to reside in an area in the $5^{\prime}$ region of the gene which contains a high degree of linkage disequilibrium. This validation supports the use of genome-wide association studies to identify genes or genomic regions that previously would not have been implicated to influence various disease traits.

\section{Acknowledgements}

We gratefully acknowledge our Amish liaisons and field workers and the extraordinary cooperation and support of the Amish community without which these studies would not have been possible. We thank Dr. Gordon Tomaselli for his thoughtful comments on the manuscript.

This work was supported by NIH research grant U01 HL72515, the University of Maryland General Clinical Research Center, Grant M01 RR 16500 and the Johns Hopkins University Bayview General Research Center (M01 RR02719), General Clinical Research Centers Program, National Center for Research Resources (NCRR), NIH, the Baltimore Veterans Administration Geriatric Research and Education Clinical Center (GRECC) and the D.W. Reynolds Clinical Cardiovascular Research Center, Johns Hopkins University. Dr. Post is supported, in part, by the Paul Beeson Physician Faculty Scholars in Aging Program.

\section{Conflict of Interest}

None.

\section{References}

1 Carter N, Snieder H, Jeffery S, Saumarez R, Varma C, Antoniades L, Spector TD: QT interval in twins. J Hum Hypertens 2000;14: 389-390.

2 Newton-Cheh C, Larson MG, Corey DC, BenjaminEJ,Herbert AG,LevyD, D'Agostino $\mathrm{RB}, \mathrm{O}^{\prime}$ Donnell CJ: QT interval is a heritable quantitative trait with evidence of linkage to chromosome 3 in a genome-wide linkage analysis: The Framingham Heart Study. Heart Rhythm 2005;2:277-284.

\footnotetext{
-3 Dekker JM, Crow RS, Hannan PJ, Schouten EG, Folsom AR: Heart rate-corrected QT interval prolongation predicts risk of coronary heart disease in black and white middle-aged men and women: the ARIC study. J Am Coll Cardiol 2004;43:565-571.

-4 Okin PM, Devereux RB, Howard BV, Fabsitz RR, Lee ET, Welty TK: Assessment of QT interval and QT dispersion for prediction of all-cause and cardiovascular mortality in American Indians: The Strong Heart Study. Circulation 2000;101:61-66.
}

\footnotetext{
5 Schouten EG, Dekker JM, Meppelink P, Kok FJ, Vandenbroucke JP, Pool J: QT interval prolongation predicts cardiovascular mortality in an apparently healthy population. Circulation 1991;84:1516-1523.

-6 Priori SG, Napolitano C: Genetics of cardiac arrhythmias and sudden cardiac death. Ann N Y Acad Sci 2004;1015:96-110.

-7 Myerburg RJ, Castellanos A: Emerging paradigms of the epidemiology and demographics of sudden cardiac arrest. Heart Rhythm 2006;3:235-239.
} 
8 Arking DE, Pfeufer A, Post W, Kao WH, Newton-Cheh C, Ikeda M, West K, Kashuk C, Akyol M, Perz S, Jalilzadeh S, Illig T, Gieger C, Guo CY, Larson MG, Wichmann HE, Marban E, O'Donnell CJ, Hirschhorn JN, Kaab S, Spooner PM, Meitinger T, Chakravarti A: A common genetic variant in the NOS1 regulator NOS1AP modulates cardiac repolarization. Nat Genet 2006;38:644-651.

9 Sorkin J, Post W, Pollin TI, O'Connell JR, Mitchell BD, Shuldiner AR: Exploring the genetics of longevity in the Old Order Amish. Mech Ageing Dev 2005;126:347-350.

10 Herbert A, Gerry NP, McQueen MB: Response to comments on 'A common genetic variant is associated with adult and childhood obesity'. Science 2007;315:e187-e188.
Almasy L, Blangero J: Multipoint quantitative-trait linkage analysis in general pedigrees. Am J Hum Genet 1998;62:1198-1211.

12 Tregouet DA, Escolano S, Tiret L, Mallet A, Golmard JL: A new algorithm for haplotypebased association analysis: The StochasticEM algorithm. Ann Hum Genet 2004;68: 165-177.

13 Jouven X, Desnos M, Guerot C, Ducimetiere P: Predicting sudden death in the population: the Paris Prospective Study I. Circulation 1999;99:1978-1983.

14 Sotoodehnia N, Siscovick DS, Vatta M, Psaty BM, Tracy RP, Towbin JA, Lemaitre RN, Rea TD, Durda JP, Chang JM, Lumley TS, Kuller LH, Burke GL, Heckbert SR: Beta2-adrenergic receptor genetic variants and risk of sudden cardiac death. Circulation 2006;113: 1842-1848.
15 Splawski I, Timothy KW, Tateyama M, Clancy CE, Malhotra A, Beggs AH, Cappuccio FP, Sagnella GA, Kass RS, Keating MT: Variant of SCN5A sodium channel implicated in risk of cardiac arrhythmia. Science 2002;297: 1333-1336.

16 Yang P, Kanki H, Drolet B, Yang T, Wei J, Viswanathan PC, Hohnloser SH, Shimizu W, Schwartz PJ, Stanton M, Murray KT, Norris K, George AL Jr, Roden DM: Allelic variants in long-QT disease genes in patients with drug-associated torsades de pointes. Circulation 2002;105:1943-1948.

17 Hong Y, Rautaharju PM, Hopkins PN, Arnett DK, Djousse L, Pankow JS, Sholinsky P, Rao DC, Province MA: Familial aggregation of QT-interval variability in a general population: Results from the NHLBI Family Heart Study. Clin Genet 2001;59:171-177. 